\title{
Saccadic movement effects on intraocular drug delivery for a wet-AMD clinical case
}

Marco Ferroni ${ }^{1}$, Matteo Cereda ${ }^{2}$, Federica Boschetti ${ }^{1}$

${ }^{1}$ Department of Chemistry, Materials, and Chemical Engineering "Giulio Natta", Politecnico di Milano (Polytechnic University of Milan), Milan, Italy; ${ }^{2}$ Department of Biomedical and Clinical Science Luigi Sacco, Sacco Hospital, University of Milan, Milan, Italy

\section{Abstract}

Nowadays, intravitreal injections are the gold standard for the treatment of age-related macular degeneration (AMD). The prediction of the transport mechanism for the injected anti vascular endothelial growth factor (anti-VEGF) is needed in order to understand its distribution and consumption after each injection. Thus, this study aims at implementing a full model of vitreous drug delivery. The main novelty of this work is the coupling between an experimental evaluation of the scleral permeability and a numerical analysis of the saccadic dependency of the transport phenomena.

Keywords: age-related macular degeneration (AMD), computational fluid-dynamics, saccade, vitreous drug delivery

\section{Introduction}

One of the main ocular diseases related to aging is the wet form of AMD, which is treated with anti-VEGF injections in the eye. This problem has been tackled with different computational approaches, considering either the influence of the tissues surrounding the posterior chamber or the saccadic movements. ${ }^{1,2}$ No work combines saccadic movements, surrounding physiological conditions, and drug injection and

Correspondence: Marco Ferroni, LaBS, Chemistry Materials and Chemical Engineering Department “Giulio Natta”, Piazza L. da Vinci 32, 20133 Milan, Italy.

E-mail:marco.ferroni@polimi.it 
consumption in a single model for a specific case of wet-AMD. Furthermore, the hydraulic conductivity behavior of the sclera has not been evaluated yet. The aim of this work is to develop a complete model of drug delivery in the posterior chamber of patients suffering of wet-AMD, showing the influence of the combination of the saccadic movements, physiological boundary conditions, and drug consumption upon drug distribution inside the vitreous.

\section{Materials and methods}

\subsection{Geometrical reconstruction, mesh, and computer code}

The idealized geometry was reconstructed from orbital magnetic resonance imaging (MRI) images, representing the effective anatomy and shape of the human posterior chamber bounded by the hyaloid membrane, the lens, and the retina-choroid-sclera (RCS) complex (Fig. 1).
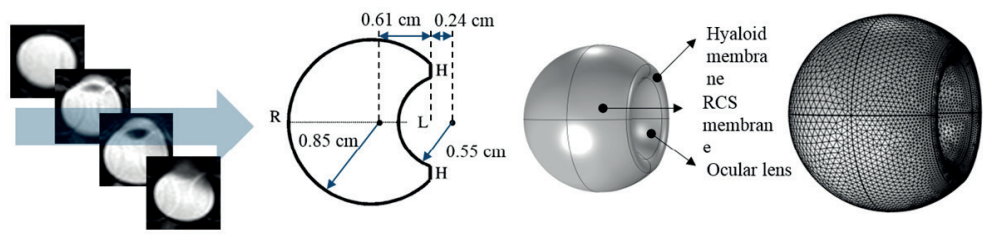

Fig. 1. MRI-based reconstruction and domain meshing.

The values of the radii of curvature of the retina and lens are equal to $0.85 \mathrm{~cm}$ and $0.55 \mathrm{~cm}$, respectively. The retina and lens have two centers of curvature with respect to the hyaloid membrane, the posterior one of $0.61 \mathrm{~cm}$, and the anterior one of 0.24 $\mathrm{cm}$. The bolus that mimics the intravitreal drug injection was modeled as a sphere with a radius of $2.29 \mathrm{~mm}$ in order to recreate the clinical injected volume in cases of wet-AMD $(0.05 \mathrm{~mL})$. Structured meshes and all the simulations were performed using the commercial software Comsol Multiphysics 5.2 (COMSOL Inc.; Burlington, MA, USA) and built with at least 250,000 elements.

\subsection{Characterization of scleral permeability}

In a similar fashion to other experimental studies in the ocular field, we used bovine samples due to their high availability and similarity to human anatomy. ${ }^{3}$ Cylindrical samples were prepared and tested within two days after slaughter and preserved at $4^{\circ} \mathrm{C}$ to slow the degradation process (Fig. 2).

The experimental setup consists of a closed chamber within which the sample is placed and constrained. The pressure drop is applied from the top and developed through a hydraulic head of saline solution that acts directly on the sample. From the bottom, the fluid flows into a graduated capillary in relation to its hydraulic con- 


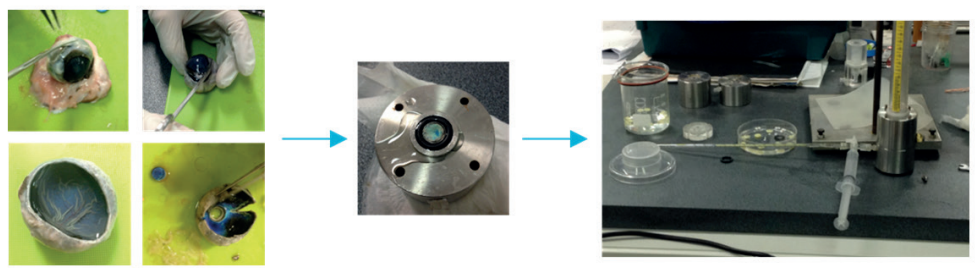

Fig. 2. Sample preparation and experimental setup for scleral permeability.

ductivity following Darcy's law, whereas its trend as function of the pressure drop is obtained through interpolation. This function is implemented in the modeled posterior chamber to describe the changes in the permeation of the vitreous humor in relation to the time changes of the pressure drop.

\subsection{Computational fluid-dynamic modeling}

The vitreous humor inside the eye was modeled as water at ocular temperature $\left(T=34^{\circ} \mathrm{C}\right)^{4}$ due to the liquefaction occurring in the presence of wet-AMD; thus, the Navier-Stokes and the continuity equations for a 3-D unsteady, incompressible Newtonian flow were solved. The imposed boundary conditions allowed us to consider the posterior chamber surrounded by its biological environment: indeed, they mimic the pressure exerted by the anterior chamber (intraocular pressure, IOP $=15 \mathrm{mmHg}$ ) and the outflow of fluid through the RCS complex, governed by Darcy's law. In particular, we imposed:

1. no aqueous permeation through the lens;

2. IOP at the posterior hyaloid surface; and

3. normal velocity across the porous RCS membrane, defined by the hydraulic conductivity function experimentally evaluated.

In addition to these conditions, the saccades were implemented. This involuntary movement during the ocular focusing of an object was defined as a fifth-grade polynomial, considering the maximum angular displacement of a saccade $\left(50^{\circ}\right)$ without the introduction of a rest time in order to represent the most stressful condition in vivo.

\subsection{Drug delivery modeling}

In order to analyze the influence of the saccadic movements on the delivery of anti-VEGF, the law of conservation of mass, Fick's law, Navier-Stokes, and continuity equations were properly coupled with each other. The imposed drug concentration of the injected bolus was equal to $0.207 \mathrm{~mol} / \mathrm{m}^{3}$, as $0.5 \mathrm{mg}$ is the standard quantity of the humanized monoclonal antibody fragment Ranibizumab, with a molecular weight of $48 \mathrm{kDa} .{ }^{5}$ The drug reaction was imposed only at the retinal surface, as shown by clinical evidence, and implemented as a linear relation between the outflow from the RCS complex and the retinal drug concentration. ${ }^{1}$ 


\section{Hydraulic Conductivity - Pressure Drop}

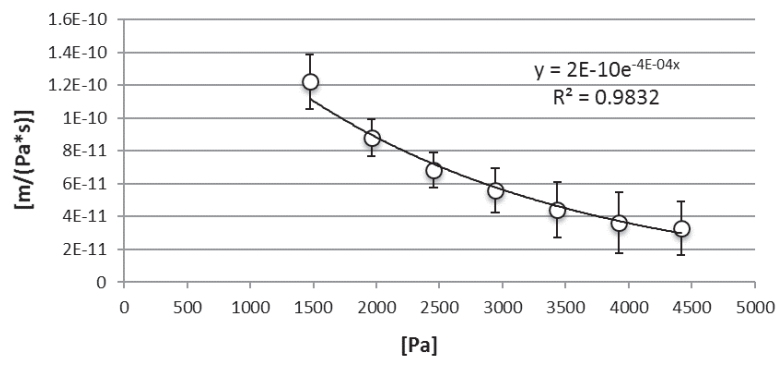

Fig. 3. Exponential decay for scleral hydraulic conductivity by varying acting pressure drop.

\section{Normalized Drug Concentration}
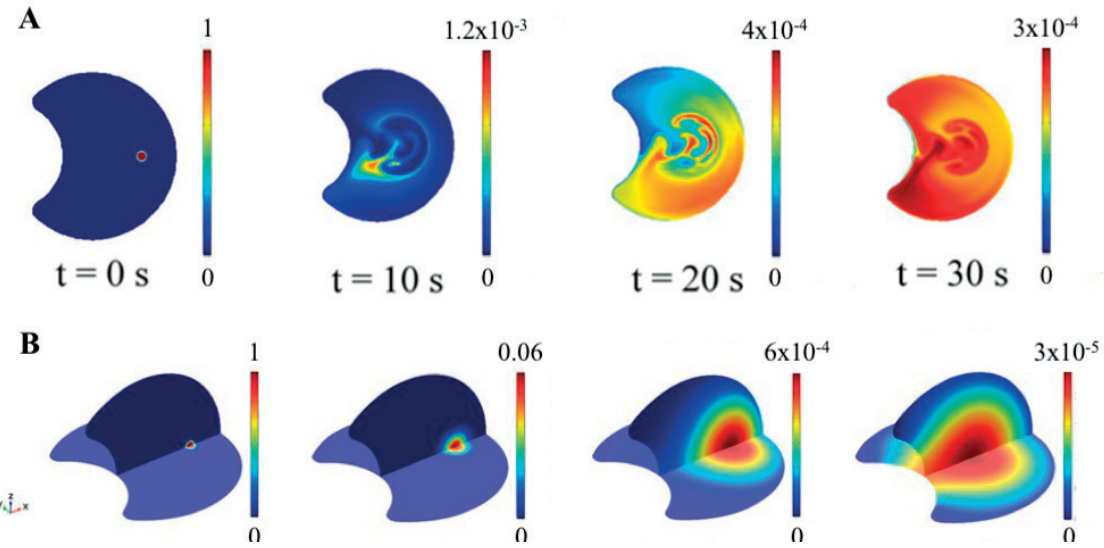

Fig. 4. Normalized concentration in the equatorial plane in case of saccade $(A)$, and in the equatorial and vertical planes without saccade $(B)$.

\section{Results}

The averaged trend of the permeability of the scleral samples related to the acting pressure drop is shown in Figure 3. The interpolation reported an exponential function, which is the typical trend for soft tissues.

The velocity distribution on the equatorial plane is purely dependent on the saccadic motion, with a slower volumetric flow rate $(2.98 \mu \mathrm{L} / \mathrm{min})$ if compared to the case without pure saccadic motion $(5.32 \mu \mathrm{L} / \mathrm{min})$, due to the mixing effect of the saccade. Streamline analysis shows two main stagnant regions on the equatorial 

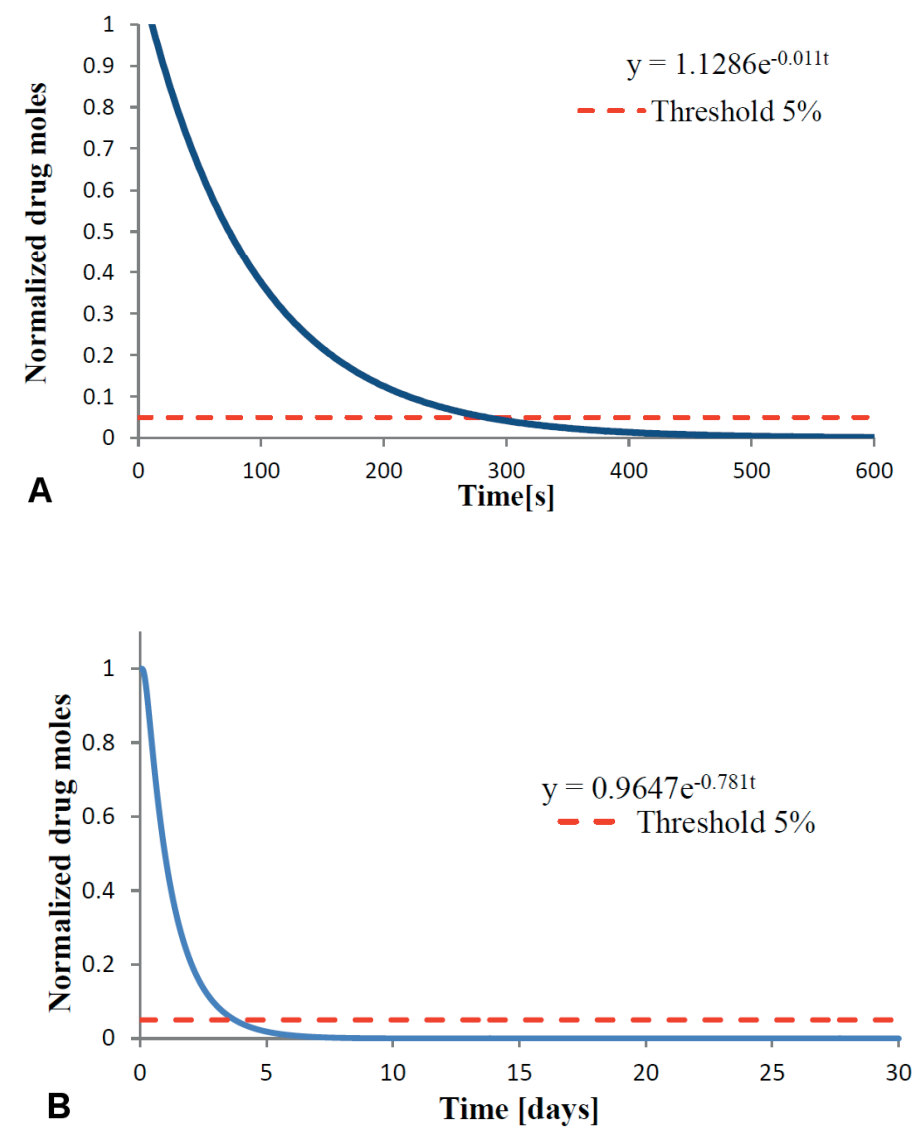

Fig. 5. Time decay of Ranibizumab in the presence $(A)$ and absence of saccade $(B)$.

plane. The distribution of the anti-VEGF in the equatorial and vertical planes, in the presence and absence of saccadic motion, is shown in Figure 4, underlining how the saccades allow a faster drug distribution. We interpolated the decay in time of the injected Ranibizumab with an exponential trend line in order to analyze the trend after the 120 seconds of simulation, imposing a threshold line of $5 \%$ under which the drug is considered totally consumed (Fig. 5A). In the absence of saccadic movements, drug uniformity is hard to reach, so its decay is considered under the $5 \%$ threshold almost four days after the injection (Fig. 5B). 


\section{Conclusion}

This study shows that saccadic movements cannot be neglected due to their great influence on both the fluid-dynamic and drug delivery mechanism inside the posterior chamber of the eye under wet-AMD conditions. The drug tends to concentrate in the stagnant zones analyzed in the fluid-dynamic part, with a pattern strictly related to the saccade before reaching a type of uniform distribution. A complete characterization of the surrounding tissues is also mandatory in order to consider the changes in permeation across the RCS complex and the specific anti-VEGF consumption near the retinal surface. The experimental evaluation of the relation between permeability and applied pressure drop as well as its implementation in a computational fluid-dynamics model represents a novelty in this field.

\section{References}

1. Stay MS, Xu J, Randolph TW, Barocas VH. Computer simulation of convective and diffusive transport of controlled-release drugs in the vitreous humor. Pharm Res. 2003;20(1):96-102. Available from:http://www.ncbi.nlm.nih.gov/pubmed/12608542

2. Modareszadeh A, Abouali O, Ahmadi GG. Saccade movements effect on the intravitreal drug delivery in vitreous substitutes: a numerical study. Biomech Model Mechanobiol. 2013;12(2):281-290. doi:10.1007/s10237-012-0398-3

3. Xu J, Heys JJ, Barocas VH, Randolph TW. Permeability and diffusion in vitreous humor: implications for drug delivery. Pharm Res. 2000;17(6):664-669.

4. Romano MR, Vallejo-Garcia JL, Romano V, Angi M, Vinciguerra P, Costagliola C. Thermodynamics of vitreoretinal surgery. Curr Eye Res. 2013;38(3):371-374. doi:10.3109/02713683.2012.745160

5. Veurink M, Stella C, Tabatabay C, Pournaras CJ, Gurny R. Association of ranibizumab (Lucentis ${ }^{\circledR}$ ) or bevacizumab (Avastin ${ }^{\circledast}$ ) with dexamethasone and triamcinolone acetonide: An in vitro stability assessment. Eur J Pharm Biopharm. 2011;78(2):271-277. doi:10.1016/j.ejpb.2010.12.018 